\title{
Visual stimulation alters local 40-Hz responses in humans: an EEG-study
}

\author{
Werner Lutzenberger ${ }^{\mathrm{a}, *}$, Friedemann Pulvermüller ${ }^{\mathrm{a}}$, Thomas Elbert ${ }^{\mathrm{b}}$, Niels Birbaumer ${ }^{\mathrm{a}, \mathrm{c}}$ \\ a Institut für Medizinische Psychologie und Verhaltensneurobiologie, Universität Tübingen, 72074 Tübingen, Germany \\ ${ }^{\mathrm{b}}$ Institut für Experimentelle Audiologie, Universität Münster, 48149 Münster, Germany \\ ${ }^{c}$ Università degli Studi, Padova, Italy
}

Received 12 August 1994; revised version received 21 October 1994; accepted 21 October 1994

\begin{abstract}
Irregular changing visual patterns and coherently moving bars were presented either in the upper or lower half of the visual field of 12 human subjects. EEG responses recorded over the occipital lobe showed an increase of $40 \mathrm{~Hz}$ spectral power when a regular pattern of moving bars appeared. This enhancement of $40-\mathrm{Hz}$ activity varied as a function of visual field presentation. Coherent stimuli in the upper visual field elicited $40-\mathrm{Hz}$ enhancement at lower occipital electrodes, while coherent stimulation in the lower visual field elicited $40-\mathrm{Hz}$ enhancement at upper occipital electrodes. These results evidence that neuronal $40-\mathrm{Hz}$ responses are a correlate of perception of coherent visual patterns in humans. Area-specific $40-\mathrm{Hz}$ responses related to visual perception can be picked up in the EEG.
\end{abstract}

Keywords: Response at $40 \mathrm{~Hz}$; Electroencephalography; Evoked spectral responses; Gamma-band; Oscillations; Visual cortex

Coherent periodic activity in numerous neurons is a candidate process for integration and binding of sensory information in the cortex [18]. When an animal's brain is stimulated by moving bars, neurons of primary and higher-order visual cortices simultaneously become active at a rate of $20 \mathrm{~Hz}$ and above (gamma band) [4,5]. In humans, simultaneous and periodic activity of numerous neurons may lead to a change in spectral power of surface potentials and biomagnetic fields recorded in the EEG and MEG $[11,16]$. The present experiment was performed to compare EEG spectral responses to irregularly changing visual patterns and to regularly moving bars.

Twelve human subjects aged 26-40 were asked to look at a fixation cross in the middle of a $48-\mathrm{cm}$ video monitor where horizontal bars were presented. The monitor was $2 \mathrm{~m}$ away from the subjects' eyes and the screen area where bars appeared was $30 \times 40 \mathrm{~cm}$. In this way, stimuli exceeded 6 degrees of horizontal and 4.5 degrees of vertical visual angle, so that the perifoveal area was almost completely stimulated. Each trial started with a baseline condition lasting for $1 \mathrm{~s}$. During the baseline condition, bars appeared at randomly selected positions at a rate of $25 \mathrm{~Hz}$, that is, bars presented for $40 \mathrm{~ms}$ appeared to jump

\footnotetext{
* Corresponding author, Tel.: +49 (0) 707129 4222; Fax: +49 (0) 7071295956.
}

to random places. After the baseline, a more regular visual pattern including moving bars appeared for $1 \mathrm{~s}$ (critical condition). Bars moving slowly downwards $(3 \%)$ were presented either in the upper, lower, left, or right half of the stimulation area and visual field. In the remaining half-field, the irregular pattern continued to appear. The average density of bars was equal in the baseline and the critical conditions $\left(10\right.$ bars $\left./ 4.5^{\circ}\right)$. In the upper and lower visual field condition, the bars on the left and right were exactly aligned (see Fig. 1). Bars in the left and right half of the visual field were separated by a gap of $0.5^{\circ}$. This was done in order to avoid intersection of bars and fixation cross. Visual field conditions followed each other in pseudo-random order. Not more than two identical conditions were allowed in direct succession. The inter stimulus interval varied between 1 and $1.5 \mathrm{~s}$. Subjects were asked not to move their eyes and to attend to the stimuli presented. They were asked not to move or blink during the experiment, but to interrupt the experiment temporarily by pressing a key if needed. The experiment consisted of three experimental blocks. In each block, 32 trials were presented. This resulted in a total of 96 trials, 24 of each visual field condition.

The EEG was recorded through $17 \mathrm{Ag} / \mathrm{AgCl}$ electrodes against $\mathrm{Fp}_{\mathrm{z}}$ of the international 10/20 system. $\mathrm{Fp}_{\mathrm{z}}$ is located at one-tenth of the nasion-inion distance posterior 

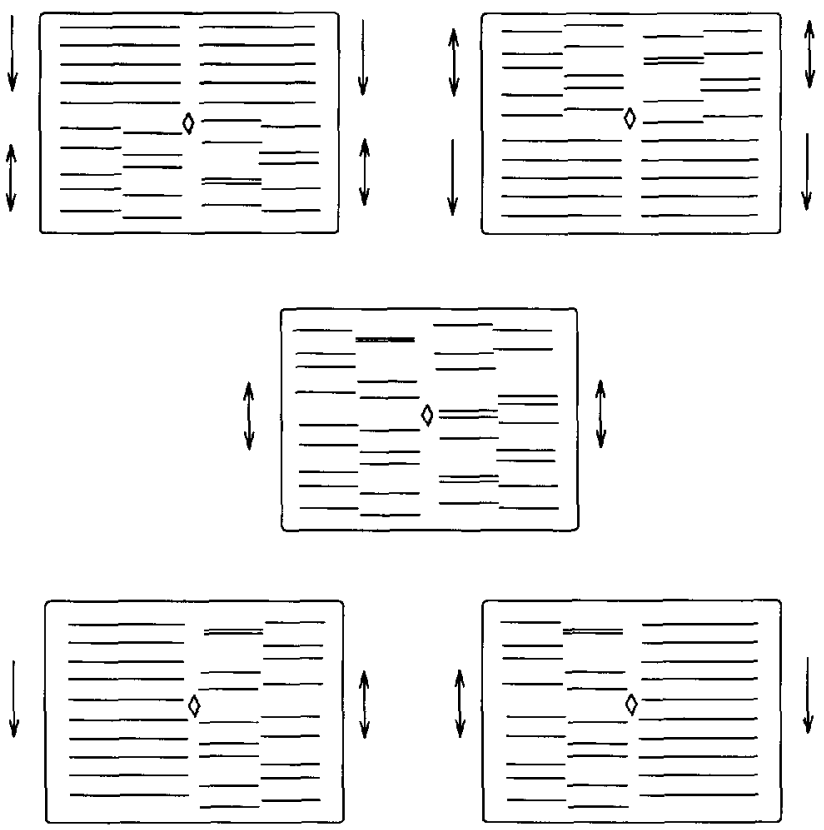

Fig. 1. Examples for five different stimulus variants used in the experiment. In the baseline condition (middle diagram), irregular patterns of bars were presented changing at a rate of $25 \mathrm{~Hz}$. In the critical conditions, bars slowly moving downwards were presented either in the upper or lower visual field (uppermost diagrams), or in the left or right visual field (lowermost diagrams). The downwards movement of the regular patterns is indicated by arrows.

to the nasion. To monitor activity over the occipital lobes, electrodes were placed in the following manner [12]. Nine electrodes were arranged in a square array $(3 \times 3$ electrodes) with $3.5 \mathrm{~cm}$ distance between next neighbors. The center electrode was placed $6 \mathrm{~cm}$ anterior to the inion. Additional electrodes were placed above and below the first and third column, as well as to the left and right of the first and third row. All distances between next neighbors were $3.5 \mathrm{~cm}$. The array is displayed in Fig. 2 . This array was selected in order to allow for current source density analysis. Signals were recorded in the $0.0796-70 \mathrm{~Hz}$ band and sampled with $200 \mathrm{~Hz}$. Trials including artifacts $>100 \mu \mathrm{V}$ caused by blinks or eye movements were excluded. The EEG was recorded for $2 \mathrm{~s}$ per trial (throughout the baseline and the critical condition).

Recording sites were projected on a sphere approximating the standard head shape. Average spherical coordinates of electrodes were calculated from Lagerlund et al. [9]. The interpolation between two adjacent projections of recording sites was based on the angle between them and the weighted sum of Legendre polynomials [15]. Next, two-dimensional spherical Laplacians were calculated and current source densities (CSDs) were estimated for the 17 recording sites $[14,15]$. CSDs were obtained for each time point of each trial. Compared to raw EEG data, CSD analysis makes it possible to calculate activity at critical electrodes independently of the activity at the reference electrode. In addition, this procedure en- hances the contribution of local brain activity to the signal, while global contributions of distant sources are minimized $[7,10]$. Note that using raw EEG data would make it impossible to determine whether spectral responses are generated at the critical electrode or at the reference, and whether they are influenced by generators distant from the electrodes. Subsequently, evoked spectral responses were calculated for three frequency bands. The procedure was the following. CSDs were first padded to zeros by using cosines, and then submitted to FFTs. Amplitudes of the FFT transformed signal were multiplied by cosine-shaped windows $(25-35 \mathrm{~Hz}, 35-45 \mathrm{~Hz}$, $62.5-77.5 \mathrm{~Hz}$ ). The filtered signals were restored by inverse FFTs and rectified by calculating root mean squares. The spectral power values were averaged over trials. Logarithms were calculated in order to approximate a normal distribution of power values. Two separate evaluations were performed. In one of them, responses to presentation of the regular pattern in the left and right visual half-fields were compared (left/right analysis). In the second evaluation, responses from the upper and the lower mode of presentation were compared (hi/lo analysis). For each condition, spectral responses obtained from $2 \times 3$ electrodes were statistically evaluated. The three

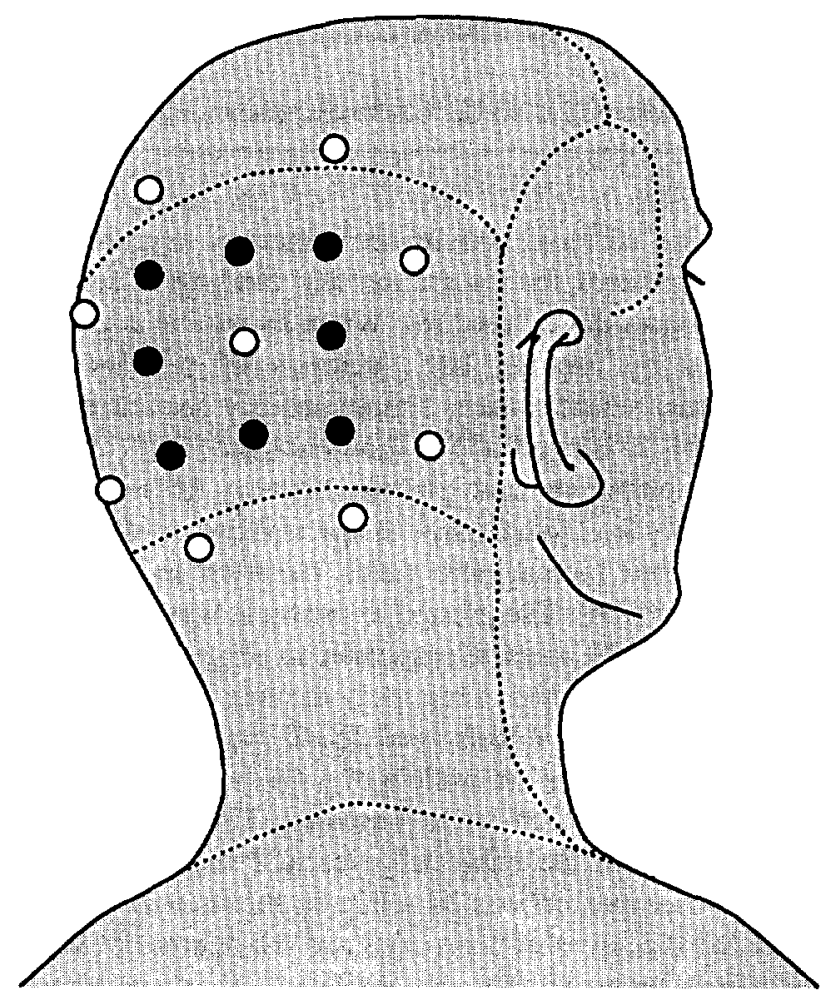

Fig. 2. Electrode array used for recording spectral responses. The center electrode was placed $6 \mathrm{~cm}$ anterior to the inion. All recordings entered CSD analyses. CSDs from the electrodes represented as black dots entered statistical analyses. Data from the three upper- versus lowermost (black) electrodes were used in the hi/lo analysis. Data from the three right- versus leftmost (black) electrodes were used in the leftright analysis. 


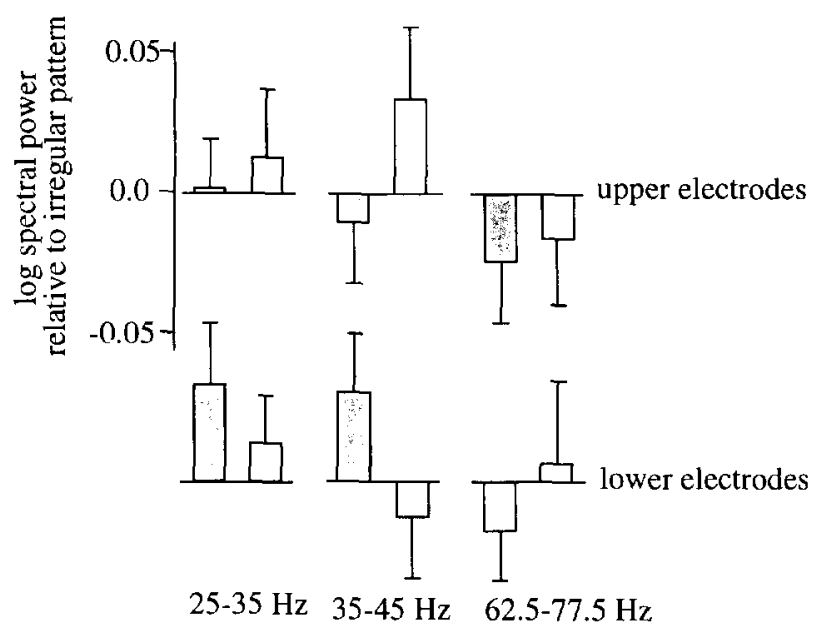

upper visual field $\square$ lower visual field

Fig. 3 Mean normalized spectral power relative to baseline in three frequency bands $(25-35 \mathrm{~Hz}, 35-45 \mathrm{~Hz}$, and $62.5-77.5 \mathrm{~Hz})$ obtained during presentation of moving bars in the upper and lower visual halffield. Displayed are mean spectral responses at the three uppermost electrodes and the three lowermost electrodes represented as black dots in Fig. 2. The middle diagrams (35-45 Hz range) display the significant interaction of presentation mode $\times$ recording site. Note that upper visual field stimulation produced greater responses at lower electrodes and conversely only in the $40-\mathrm{Hz}$ band.

electrodes of the first and third column of the $(3 \times 3)$ array were selected for the left/right-evaluation (see black dots in Fig. 2). Data from the three electrodes in the first and third row entered the hi/lo evaluation. Mean values from the first $500 \mathrm{~ms}$ of the critical condition were normalized by subtraction of the mean obtained in the first $500 \mathrm{~ms}$ of the baseline condition. Data from individual subjects averaged over trials were submitted to ANOVAs with the factors electrode (1st, 2nd, 3rd), recording site (left/right or hi/lo), and presentation mode (again, left/right or hi/lo).

Spectral responses to bars presented in the upper or lower visual fields altered spectral responses in the 35$45 \mathrm{~Hz}$ band exclusively. Effects elicited by aligned moving bars in the upper versus lower visual field were reliably different. Analysis of normalized means revealed a significant interaction of the factors recording site and presentation mode in the $35-45 \mathrm{~Hz}$ range $(F(1,11)=6.8$, $P<0.025)$. This interaction was due to stronger $40-\mathrm{Hz}$ responses at the lower electrodes in the upper visual field condition and to stronger responses at the upper electrodes in the lower visual field condition (Fig. 3). Analyses of all other frequency bands failed to reveal significant main effects or interactions. There were also no reliable differences after stimulation with short bars in the left versus right visual field condition $(F<1)$.

Two additional analyses were performed to confirm the recording site $\times$ presentation mode interaction in the $40-\mathrm{Hz}$ range: In one analysis, moving averages of spectral power were obtained in $200-\mathrm{ms}$ wide windows. Windows were shifted by $40 \mathrm{~ms}$. For each trial, the maximum value observed during presentation of the critical (regular) visual pattern entered the analysis, after subtraction of the respective baseline value (analysis of normalized maxima). Raw maximum values entered another analysis (analysis of raw maxima). In both of these analyses, the interaction of presentation mode and recording site was significant $\quad(F(1,11)=9.9, \quad P<0.01 ; \quad F(1,11)=11.6$, $P<0.01$ ). Higher $40-\mathrm{Hz}$ maxima were found at lower electrodes when the regular pattern appeared at the top, while regular patterns in the lower visual half-field elicited higher $40-\mathrm{Hz}$ maxima at upper recording sites. This is in agreement with the results from the analysis of normalized means.

The main finding of this study is that scalp topography of $40-\mathrm{Hz}$ responses shifts appropriately with stimulus position when coherent stimuli appear in the upper versus lower visual field. Stimuli appearing in the upper visual field are projected to the lower half of the retina from where information flows to more ventral (lower) sites of the primary visual cortex; stimulus information from the lower visual field is transferred to the upper half of the retina which projects to more dorsal (upper) occipital cortices [13]. Most likely, changes in evoked EEG responses go back to EPSPs in many apical dendrites of pyramidal cells in the upper cortical layers of cortical gyri (causing radial dipoles), rather than to activity in sulci (leading to tangential dipoles) [2]. Because large parts of the human striate cortex are buried in the calcarine fissure and in the interhemispheric sulcus, it is possible that the local change of $40-\mathrm{Hz}$ power is primarily generated in primary visual cortices outside these sulci. However, neurons sensitive to moving bars are very well known to be present not only in primary cortices, but also in higherorder visual cortices [8]. It has even been shown that synchronized periodic activity can be observed in primary and higher-order visual cortices of animal brain, when moving bars are presented [5]. Therefore, neural activity in higher-order visual cortices may have contributed to the observed changes in EEG spectral responses. In addition, specific changes in the $40-\mathrm{Hz}$ band of the EEG signal must be due to simultaneous activity of large neuronal populations close to the recording sites, otherwise the signal would not be strong enough to spread to the electrodes. These numerous neurons are unlikely to be restricted to small cortical areas, rather they are likely to be distributed over several areas. Finally, the fact that differential $40-\mathrm{Hz}$ power changes could be recorded at up to three electrodes up to $7 \mathrm{~cm}$ apart also argues that distributed networks led to the observed responses. Therefore, it may well be that assemblies of strongly coupled neurons distributed over various visual cortices of both hemispheres are the generator of $40-\mathrm{Hz}$ power changes visible in the EEG. Such neural assemblies may 'oscillate' simultaneously in the $40-\mathrm{Hz}$ range after stimulation with a behaviorally significant visual stimulus. However, differen- 
tial spectral responses may also result from more complex spatio-temporal patterns of neuronal activity to which large numbers of neurons contribute [1]. It is, however, important to note that coherent oscillations or repetitive spatio-temporal patterns of activity are only possible in strongly coupled cell assemblies [3].

There were no $35-45 \mathrm{~Hz}$ differences in the spectral responses to moving bars presented in the left versus right visual field. Possible reasons include shorter bar length (see Fig. 1), and the failure to stimulate mirror image areas in the two hemispheres. Interhemispheric circuits could be important for generating $35-45 \mathrm{~Hz}$ activity [6] and the greater cancellation of visually evoked responses by our CSD analysis when this activity is asymmetrical could have increased sensitivity to other activity in the hi/lo analysis.

Our results require that: (i) the assemblies responsible for elevated $35-45 \mathrm{~Hz}$ activity be large enough to cause a detectable change at the surface of the skull; and (ii) their cortical topography vary with visual field stimulus location. These assemblies appear to span cortical areas in both hemispheres. Our findings are furthermore in agreement with results reported earlier that behaviorally significant, common and meaningful real words lead to stronger gamma-band responses than behaviorally irrelevant, uncommon and meaningless pseudowords [11]. It appears that the brain expresses its opinion about behavioral relevance of stimuli in terms of gamma-band responses [17].

Supported by the Deutsche Forschungsgemeinschaft (DFG, SFB 307/B 1 and Pu 97/2) and by the Bundesministerium für Forschung und Technologie (BMFT, Helmholtz-Fellowship to FP).

[1] Abeles, M., Corticonics - Neural Circuits of the Cerebral Cortex, Cambridge University Press, Cambridge, 1991.

[2] Birbaumer, N., Elbert, T., Canavan, A.G.M. and Rockstroh, B., Slow potentials of the cerebral cortex and behavior, Physiol. Rev., 70 (1990) 1-41.

[3] Braitenberg, V., Cell assemblies in the cerebral cortex. In R. Heim and G. Palm (Eds.), Theoretical Approaches to Complex Systems.
(Lecture Notes in Biomathematics, Vol. 21), Springer, Berlin, 1978, pp. 171-188.

[4] Eckhorn, R., Bauer, R., Jordan, W., Brosch, M., Kruse, W, Munk, M. and Reitboeck, H.J., Coherent oscillations: a mechanism of feature linking in the visual cortex? Multiple electrode and correlation analysis in the cat, Biol. Cybernet., 60 (1988) 121-130.

[5] Engel, A.K., König, P., Kreiter, A.K., Schillen, T.B. and Singer, W., Temporal coding in the visual cortex: new vistas on integration in the nervous system, Trends Neurosci., 15 (1992) 218-226.

[6] Engel, A.K., König, P., Kreiter, A.K. and Singer, W., Interhemispheric synchronization of oscillatory neuronal responses in cat visual cortex, Science, 252 (1991) 1177-1179.

[7] Hjorth, B., An on-line information of EEG scalp potentials into orthogonal source derivations, Electroencephalogr. Clin. Neurophysiol., 39 (1975) 526-530.

[8] Hubel, D., Eye, Brain, and Vision, Freeman, New York, 1988.

[9] Lagerlund, T.D., Sharbrough, F.W., Jack Jr., C.R., Erickson, B.J., Strelow, D.C., Cicora, K.M. and Busacker, N.E., Determination of 10-20 system electrode locations using magnetic resonance scanning with markers, Electroencephalogr. Clin. Neurophysiol., 86 (1994) 7-14.

[10] Law, S.K., Rohrbaugh, J.W., Adams, C.M. and Eckhardt, M.J., Improving spatial and temporal resolution in evoked EEG responses using surface Laplacians, Electroencephalogr. Clin. Neurophysiol., 88 (1993) 309-322.

[11] Lutzenberger, W., Pulvermüller, F. and Birbaumer, N., Words and pseudowords elicit distinct patterns of $30-\mathrm{Hz}$ activity in humans, Neurosci. Lett., 176 (1994) 115-118.

[12] Maier, J., Dagnelie, H., Spekreijse, H. and Van Dijk, B.W., Principal component analysis for source localization of VEPs in man, Vision Res., 27 (1987) 165-177.

[13] Mason, C. and Kandel, E.R., Central visual pathways. In E.R. Kandel, J.H. Schwartz and T.M. Jessell (Eds.), Principles of Neural Science, Vol. 3, Prentice Hall, London, 1994, pp. 420-439.

[14] Perrin, F., Comments on article by Biggins et al., Electroencephalogr. Clin. Neurophysiol., 83 (1992) 171-172.

[15] Perrin, F., Pernier, J., Bertrand, O. and Echallier, J.F., Spherical splines for scalp potential and current source density mapping, Electroencephalogr. Clin. Neurophysiol., 72 (1989) 184-187.

[16] Pfurtscheller, G. and Neuper, C., Simultaneous EEG $10 \mathrm{~Hz}$ desynchronization and $40 \mathrm{~Hz}$ synchronization during finger movements, NeuroReport, 3 (1992) 1057-1060.

[17] Pulvermüller, F., Preissl, H., Eulitz, C., Pantev, C., Lutzenberger, W., Elbert, T. and Birbaumer, N., Brain rhythms, cell assemblies, and cognition: evidence from the processing of words and pseudowords, Psycoloquy, 5(48) (1994).

[18] Singer, W., Putative functions of temporal correlations in neocortical processing. In C. Koch and J. Davis (Eds.), Large Scale Neuronal Theories of the Brain, MIT Press, Boston, MA, 1994. 